\title{
DETECTING ANTARCTIC SEALS AND FLYING SEABIRDS BY UAV
}

\author{
Osama Mustafa ${ }^{1}$, Christina Braun ${ }^{2}$, Jan Esefeld ${ }^{1,2}$, Stefan Knetsch ${ }^{1}$, Jakob Maercker ${ }^{1}$, Christian Pfeifer ${ }^{1}$, Marie-Charlott Rümmler ${ }^{1}$ \\ ThINK - Thuringian Institute of Sustainability and Climate Protection, Germany (osama.mustafa@think-jena.de, \\ stefan.knetsch@think-jena.de, jakob.maercker@think-jena.de, christian.pfeifer@think-jena.de, marie-charlott.ruemmler@think- \\ jena.de); ${ }^{2}$ Institute of Ecology and Evolution, Friedrich Schiller University Jena, Germany (jan.esefeld@uni-jena.de, \\ chr.braun@uni-jena.de)
}

KEY WORDS: UAV, Birds, Seals, Antarctic, Wildlife, Mapping, Monitoring, Detection

\begin{abstract}
:
In recent years Unmanned Aerial Vehicles (UAVs) became a fast-developing technology with manifold fields of application. In the field of wildlife biology, it offers the opportunity to quantify populations, to map the spatial distribution of species and to observe the behavior of animals with no or low disturbance. Moreover, UAV based mapping allows to obtain data from sites which are hardly or not accessible and to cover much larger areas than by traditional ground based methods. The advantages of UAV based mapping are of particular relevance under the harsh conditions of Antarctic fieldwork. Whether certain species qualify for UAV based monitoring depends on their detectability from the distance and the distinctiveness of their characteristics in comparison to other species, which has not been studied for Antarctic species in detail so far. This study aims to evaluate how and under which conditions, particularly flight height, Antarctic flying seabird and seal species are detectable in aerial imagery. A trial was conducted comparing the detection rate of different observers for several Antarctic species in aerial images of different ground sample distances. Descriptions of individual appearance as well as body size dimensions are delivered for all species. For most of the investigated species, monitoring proves to be possible from practical flight heights, while others are still very hard to detect even in low altitudes. A concluding table is given aiming to provide a guide for future surveys on which flight altitudes to chose and how to identify focal study objects.
\end{abstract}

\section{INTTRODUCTION}

\subsection{Introduction}

Changes in Antarctic wildlife populations are difficult to monitor. Seals can be found at almost all parts of the Antarctic coast. Breeding sites of flying seabirds are often located at remote and difficult to access locations. Therefore, by ground based classical methods a high effort is necessary to conduct detailed surveys of larger areas with often incomplete results. Besides, mapping or counting these populations on ground implies a significant disturbance of the animals (Pfeiffer, 2005; Schuster, 2010). Thus, the use of UAVs can provide an alternative to map colonies and aggregations of seabirds and seals. It has been shown for different species that counting individuals on very high resolution aerial images generated by UAV can give more accurate results then counting on ground (Hodgson et al., 2016; Hodgson et al., 2018; Adame et al., 2017). There are various studies available that focus on the comparison between ground counting and aerial images by UAV for birds and seals (Walker, 2014; Chabot et al., 2015; Adame et al., 2017; Hodgson et al., 2018). In the Antarctic, Goebel et al. (2015) compared counts of Gentoo- and Chinstrap Penguins on ground with counts from UAV-generated images. Mustafa et al. (2017) used UAVs for monitoring penguin colonies to distinguish between different penguin species and examined the potential of different sensors for penguin detection (Thermal, Ultraviolet, Near-IR and RGB). KorczakAbshire et al. (2018) used images recorded by a fixed-wing UAV to count four species of birds (Adélie- and Chinstrap Penguins, Southern Giant Petrels and Antarctic Shags) and two species of seals (Southern Elephant Seals and Weddell Seals). Borowicz et al. (2018) combined UAV and satellite imagery with ground-based data for a multi-modal survey of Adélie Penguin colonies. The estimation of body mass and body conditions of Leopard Seals by using photogrammetry on UAV images was successfully performed by Krause et al. (2017). The state of knowledge about the response of wildlife to UAV systems is concluded in Mustafa et al. (2018).

However, the questions of how different species can be identified and differentiated operationally and how efficient flight planning can be combined with high recognition quality have received little attention so far.

This study investigates the detectability of five seal and six flying seabird species in the maritime Antarctic by UAVs. Typical features are emphasized and the recognizability by aerial image analysis is investigated. To support the flight planning process, the ground sample distance (GSD) necessary for a reliable recognition of the respective species will be examined.

\section{METHODS}

\subsection{Study Area and selected species}

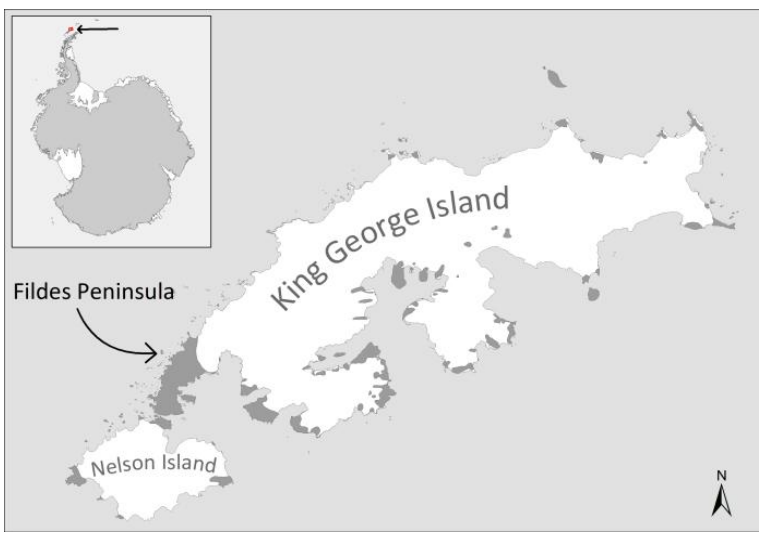

Figure 1. Location of the study site 
The study was conducted on Fildes Peninsula (King George Island, South Shetland Islands, maritime Antarctic, Figure 1) during the austral summer seasons (October - February) 2013/14, 2014/15, 2016/17 and 2017/18.

In this study six species of birds and five species of seals (Table 1) were examined. Brown and South Polar Skuas were not distinguished and treated as one species due to their similar appearance and cross-over of characteristics. The penguins of the genus Pygoscelis, which are frequently found in the area, were not taken into account, as they have already been dealt with in detail in previous studies (Perryman et al., 2014; Ratcliffe et al., 2015; Mustafa et al., 2017; Korczak-Abshire et al., 2018).

\begin{tabular}{|c|c|}
\hline Seals & Birds \\
\hline Antarctic Fur Seal & Antarctic Shag \\
Arctocephalus gazella & Phalacrocorax atriceps \\
\hline Crabeater Seal & Antarctic Tern \\
Lobodon carcinophagus & Sterna vittata \\
\hline Leopard Seal & Kelp Gull \\
Hydrurga leptonyx & Larus dominicanus \\
\hline Southern Elephant Seal & Skua \\
Mirounga leonina & Catharacta spec. \\
\hline Weddell Seal & Snowy Sheathbill \\
Leptonychotes weddelli & Chionis alba \\
\hline & Southern Giant Petrel* \\
& Macronectes giganteus \\
\hline
\end{tabular}

Table 1. Bird and seal species examined in this study. *differentiated in adult and juvenile

\subsection{UAV flights and Sensor Specifications}

The images analyzed in this study were acquired with three different UAV each equipped with a different camera (Table 2). It came to use an octocopter UAV 'Mikrokopter MK" with a 'Samsung MX500' and a 'EX-W20NB' lens, a quadrocopter UAV 'DJI Phantom 4 pro' with the original DJI camera and a fixed wing UAV 'Bormatec Ninox' with a 'MAPIR Survey-2 RGB'.

\begin{tabular}{|l|l|l|c|}
\hline Camera & $\begin{array}{l}\text { Image } \\
\text { resolution } \\
\text { [pixel] }\end{array}$ & $\begin{array}{l}\text { Sensor } \\
\text { width } \\
\text { [mm] }\end{array}$ & $\begin{array}{l}\text { Focal } \\
\text { length } \\
\text { [mm] }\end{array}$ \\
\hline $\begin{array}{l}\text { Samsung MX500 + } \\
\text { EX-W20NB lens }\end{array}$ & $6480 \times 4320$ & $23.5 \times 15.7$ & 20 \\
\hline $\begin{array}{l}\text { 1" CMOS 20MP (DJI } \\
\text { Phantom 4pro) }\end{array}$ & $5472 \times 3648$ & $13.2 \times 8.8$ & 8.8 \\
\hline MAPIR Survey-2RGB & $4608 \times 3456$ & $6.17 \times 4.63$ & 3.97 \\
\hline
\end{tabular}

Table 2. Cameras used in this study

Flights were conducted with a ground speed of $6-15 \mathrm{~m} / \mathrm{s}$ at a flight height of $30-150 \mathrm{~m}$ AGL in a grid pattern with at least $60 \%$ image overlap between and within a flight path. Depending on the flight distance about $50-1.000$ images in JPEG format were taken per flight. Orthomosaics were created using the photogrammetry software Agisoft PhotoScan Pro.

\subsection{Detection Characteristics}

Detection characteristics comprise measurements and descriptions of individuals of the different species in the way they appear on UAV images. The maximum width and length of the visible body for 20-25 individuals of five species of seals and five species of birds were measured in orthomosaics using ESRI ArcGIS 10.5 (ESRI, 2016). These measurements do not only rely on the traditionally measured morphological body dimensions but also on the posture of the animal (e.g. neck outstretched frontal or pulled back) and the angle of view of the camera lens. As seals at the beach often have a twined posture, the length along the centre-line of their visible shape was measured. By visually analyzing the orthomosaics it was found that colonies of Antarctic Shags are much clearer identifiable by their nest structures than by body shape. Therefore, the parameters nest diameter and nest distance (center to center) to detect and quantify breeding sites of this species were used. Juvenile Southern Giant Petrels were excluded from the measurement since their size depends on age and thus on the date of the UAV flights. The description of the appearance of the different species at UAV images were composed from the impressions of different experienced observers.

\subsection{Appropriate Ground Sample Distance (GSD)}

For planning UAV mapping campaigns flight height is a crucial parameter. Low flight heights provide a small ground sample distance (GSD), however, cover a smaller area, as the flight paths need to be closer together to ensure a sufficient overlap of the single images. A mapping at higher flight heights allows covering a larger area but the resulting orthomosaic will have a larger GSD as well. The relation of GSD and flight height above ground level (AGL) depends on camera specification (sensor size, image size, focal length) as described by (1). Table 3 shows this relation for the cameras used in this study.

$$
G S D=\frac{h * s w}{f * i w}
$$

where $\quad \mathrm{GSD}=$ ground sample distance $[\mathrm{mm} / \mathrm{pix}]$

$\mathrm{h}=$ flight height AGL [mm]

$\mathrm{sw}=$ sensor width $[\mathrm{mm}]$

$\mathrm{f}=$ focal length $[\mathrm{mm}]$

iw = image width [pix]

\begin{tabular}{|l|c|c|c|c|c|}
\hline Flight height [m AGL] & $\mathbf{2 5}$ & $\mathbf{5 0}$ & $\mathbf{7 5}$ & $\mathbf{1 0 0}$ & $\mathbf{2 0 0}$ \\
\hline GSD (Samsung) [mm] & 4.5 & 9.1 & 13.6 & 18.1 & 36.3 \\
\hline GSD (DJI) [mm] & 6.8 & 13.7 & 20.6 & 27.4 & 54.8 \\
\hline GSD (Mapir) [mm] & 8.4 & 16.9 & 25.3 & 33.7 & 67.5 \\
\hline
\end{tabular}

Table 3. Ground sample distance (GSD) at different flight

heights above ground level (AGL) for the three cameras used

To test for the detectability of different species at different GSDs, an observer trial was applied. Each species was represented by 1-26 individuals in three different images, which were provided in several GSDs. In some cases, two species were pictured on the same image. Additionally, three dummy images, plus the respective replicas, without any birds or seals, but different environmental characteristics were in the image pool.

For each species, 5 to 6 different GSD levels were chosen. The smallest GSD was defined as a ground resolution sufficient to clearly recognize the individual animals by an experienced observer who is familiar with the respective species. The largest GSD was chosen with resolutions where the same observer was not able to detect individuals anymore, or at the limits of practicability (i.e. according flight heights higher than maximal suitable flying altitudes). The GSD of the 3 or 4 images in between were chosen in an equal manner. For a better comparison of the species, one GSD was chosen to appear in all species: $30 \mathrm{~mm}$. This GSD was also chosen due to its practicality in field work since it implies a relatively useful flight altitude of about $100 \mathrm{~m}$ (Table 3). This altitude provides a 
good compromise between covered area, ground resolution and potential disturbance effects (Rümmler et al., 2018) and is commonly used with the equipment regarded here. Resulting from these considerations, the following GSDs were used for the test (Table 4).

\begin{tabular}{|l|r|r|r|r|r|r|}
\hline & GSD1 & GSD2 & GSD3 & GSD4 & GSD5 & GSD6 \\
\hline Dummy images & 20 & 30 & 40 & 60 & 80 & \\
\hline Antarctic Fur Seal & 30 & 86 & 142 & 198 & 254 & 310 \\
\hline Crabeater Seal & 30 & 100 & 170 & 240 & 310 & \\
\hline Leopard Seal & 30 & 100 & 170 & 240 & 310 & \\
\hline S. Elephant Seal & 30 & 100 & 170 & 240 & 310 & \\
\hline Weddell Seal & 30 & 100 & 170 & 240 & 310 & \\
\hline Antarctic Shag & 15 & 30 & 60 & 90 & 120 & 150 \\
\hline Antarctic Tern & 7 & 18 & 30 & 41 & 52 & \\
\hline Kelp Gull & 16 & 30 & 44 & 58 & 72 & \\
\hline Skua & 17 & 30 & 43 & 56 & 69 & \\
\hline Snowy Sheathbill & 20 & 30 & 40 & 60 & 80 & \\
\hline S. Giant Petrel (ad) & 10 & 30 & 50 & 70 & 90 & \\
\hline S. Giant Petrel (juv) & 10 & 30 & 50 & 70 & 90 & \\
\hline
\end{tabular}

Table 4. Overview over the GSD levels [mm] used for the observer test.

To simulate the image anti-aliasing of the camera (Savazzi, 2011) a $7 \times 7$ Gaussian Low Pass filter was applied to the replicas. Each image represented a section of $20 \times 20 \mathrm{~m}$. The GSD of the replicas was obtained by resampling (cubic convolution) the originals.

All in all seven observers had to identify individuals of the different species. Three of the observers have also been responsible for providing the images and were therefore excluded from counting the species of the respective images and regarded as a 'composite' observer. This way the number of observers adds up to six, hereafter called "theoretical observers". All observers are experienced with analyzing aerial images and know the species from own fieldwork at the study site. Observers had to note the recognized number of individuals for each species in every image.

For seals, additionally the "seal" category was implemented which included all seals species. This was done because of the similar appearance of some seal species and to examine the opportunity to count seals when the species are not distinguishable. In total the number of images to observe was 186. The images were viewed by each observer in an identical ArcGIS document (ESRI, 2016) in a fixed order. The order was randomized with the constraint that replicas with a large GSD (coarse ground resolution) were placed before those with a small GSD (fine ground resolution).

Statistical analyses and compilation of graphs were accomplished in R (R Core Team 2018).

\section{RESULTS}

\subsection{Detection Characteristics}

\subsubsection{Seals}

Body size measurements revealed that most of the seal species lay in a similar range of length and width (Figure 2). Only Antarctic Fur Seals are clearly separated by size from all other seals. For large individuals of Elephant Seals a clear identification is also possible as none of the other species reaches these large sizes. The specific characteristics of all species will be reviewed hereafter, together with appearance characteristics (Appendix).
The Antarctic Fur Seal is the smallest seal in Antarctica (measured length ca. 1-1.5 m) with a relative colorful brown, blonde, black or grey fur. The hind flippers are often rotated forward and thus not visible. Their shape varies as they often raise the torso.

Crabeater Seals are unicolored. The color varies between brown, blonde, creamy, grey and silver-white. They usually appear brighter than other seals and their body is relatively slender.

The Leopard Seals are grey with an almost black dorsum. They have a large head, are usually stretched out and appear a little stiff - only slightly bended. The shape is long stretched, cigarlike. Their preferred rest places are at ice floes where often the typical red colored excrements can be found close to the resting seals.

The Southern Elephant Seal is variable in color with a dark to pale grey or brown fur which is blotchy during moult. They have a relatively rotund shaped body and the head end often appears cigar-shaped pointed. Remarkable is the relatively strong sexual dimorphism which is expressed in the fact that mature males are larger (measured length up to $4.42 \mathrm{~m}$ ) than the females and also larger than all other seals. Often Elephant Seals lie closely together in groups.

Weddell Seals have a grey fur which is brighter at the ventral area. They have a relatively small head and flippers. The body shape is rotund. Individuals often lie with bend posture.
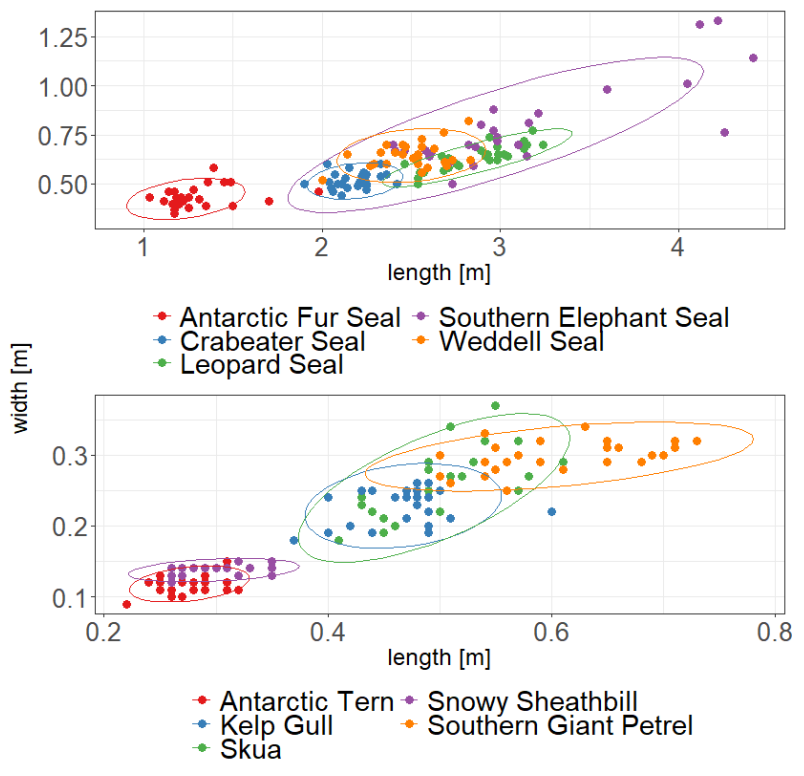

Figure 2. Body size measurements of seals (above) and birds (below). Ellipses indicate 95\% confidence level.

\subsubsection{Birds}

Measurements of size revealed two clearly separated groups within bird species (Figure 2). Antarctic Terns and Snowy Sheathbills, as the smallest species in the study, have a clear overlap but are separated from the three other species. The largest species is the Southern Giant Petrel, large individuals are clearly distinguishable from other bird species at the site. For smaller individuals an overlap occurs with Skuas and larger individuals of Kelp Gull. Those two themselves have a considerable overlap in length and width.

Adult Antarctic Shags are black with the exception of the white belly and white side of their long, narrow neck which is visible when stretched out. The shape of the individuals appears diverse and irregular. The juveniles have a brown plumage and are hardly distinguishable from the brown colored nests. They breed in colonies at rock or cliffs often close to penguin 


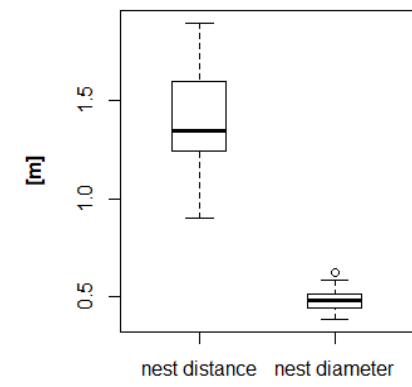

Figure 3. Measurements of nest distance and nest diameter for Antarctic Shag colonies. As this species is better to detect by nests than by individuals, Antarctic Shags were not included in the comparison of Figure 2. The nests are cup-shaped, surrounded by star shaped guano stains and have a distance of $1.25-1.65 \mathrm{~m}$. The measurements of nest

dimensions are displayed in Figure 3.

The Antarctic Tern has a greyish and white back with a

black cap and a deeply forked tail. They were found breeding in loose groups at flat areas with bright pebbles that were about their size $(0.25-0.3 \mathrm{~m})$. At these areas they were very hard to find but good to identify once found.

Kelp Gulls have a white head and tail as well as a slate black back which is often interrupted by white marks close to the tail. Well recognizable is the curved boundary between head and back. They breed in small colonies at rocks or cliffs close to the coast.

The well camouflaged Skuas are heterogeneous in color (greyish-brown) and pattern though their head and tail appear mostly darker that the back. They nest individually at a distance of about $100 \mathrm{~m}$ at high grounds.

Snowy Sheathbills have an entirely white plumage with shades of grey and a clearly distinct drop shape. They were found in or close to penguin colonies and occurred there in small groups.

The adult Southern Giant Petrel appears mostly heterogeneous greyish-brown with an often brighter or white head, neck and breast. There are also completely dark or white morphs. Distinctive is the large pale yellowish bill. Juveniles are grey with a lighter head and their shape appears roundish with fluffy edges. Southern Giant Petrels breed in small colonies at higher ground close to the coast.

\subsection{Appropriate Ground Sample Distance (GSD)}

In total, the number of individuals to detect on all images (including all GSDs) was 5,676 (946 per observer). The six observers detected all in all 3,519 individuals (62\%).

When comparing the individual detection rates of the observers over all species and GSDs, only the observer with the best detection rate (here: observer 5), and the observer with the worst detection rate (here: observer 6) proved to be statistically different (Figure 5 and Table 5).

The detection rate was determined for all species separately to investigate at which GSD species will be clearly detectable. Figure 4 shows the results of the trial. As can be seen, the detection curve varies for most of the species, which is why they will be viewed separately in the following section.

Antarctic Fur Seals were detectable at GSDs of $198 \mathrm{~mm}$ and below. With coarser ground resolutions no observer was able to find any Fur Seals. From this GSD on, the detection rate improves further on with a relatively low deviation and ends at a $100 \%$ detection score for the smallest GSD of $30 \mathrm{~mm}$. All observers were able to detect the correct number of Fur Seals in all images at this resolution.

For Crabeater Seals, the detection was already possible for some observers at the large GSD of $310 \mathrm{~mm}$. However, even at the finest tested resolution ( $30 \mathrm{~mm}$ GSD) only about $75 \%$ of the individuals were correctly detected. In all GSDs, the deviation was high, implicating a strong uncertainty between observers and images.
Leopard Seals showed an almost linear increase in detectability with decreasing GSD. The deviation was relatively high in the medium GSDs, but lower for the largest/smallest GSD.

For Southern Elephant Seals, the detection rate stayed at a very low level for the two largest GSDs, but then increased strongly with decreasing GSD. As for fur seals, in the finest ground resolution the detection rate reached $100 \%$ with a very low uncertainty of the observers. For all GSDs, the deviation was very low.

The lowest detection rate of all seals was found in Weddell Seals, with no correct detections at GSDs of $170 \mathrm{~mm}$ and above for all observers and even at the lowest GSD only a detection rate of about $60 \%$. The deviation was relatively low in all GSDs.
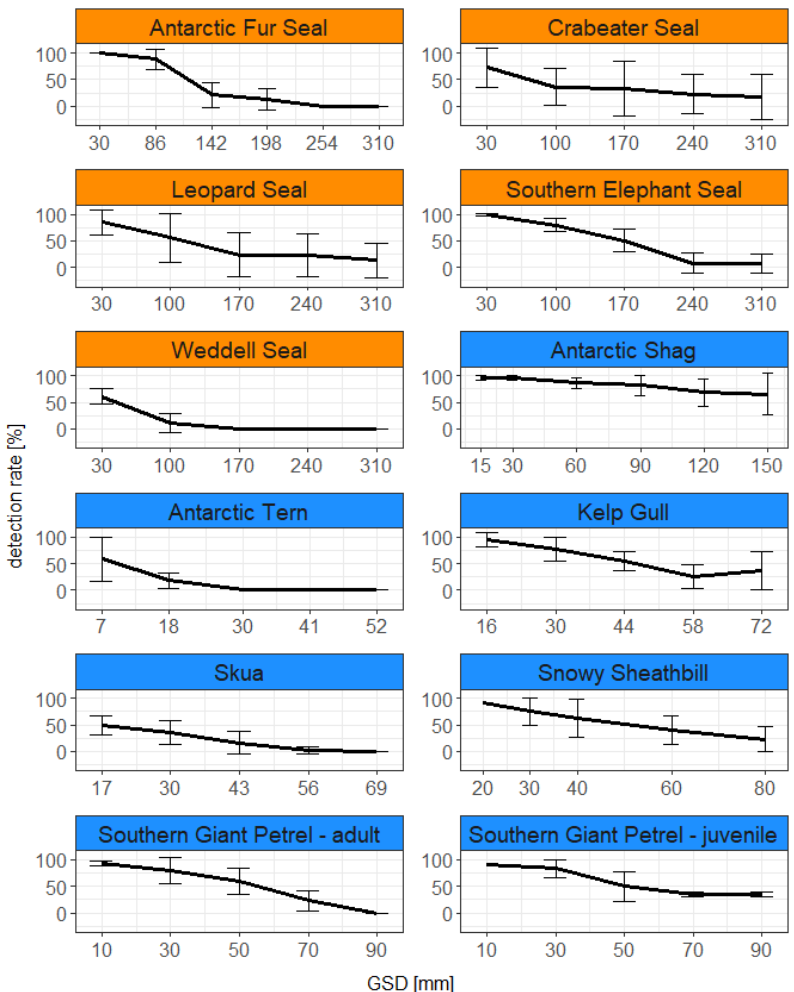

Figure 4. Mean detectability and uncertainty of all species at different GSD with whiskers representing one standard deviation above and below the mean

In bird species, Antarctic Shag nests had the best detection rates: even at $120 \mathrm{~mm}$ GSD, already more than $60 \%$ of the nests were detected. At $30 \mathrm{~mm}$ GSD the highest detection rate of almost $100 \%$ was reached and maintained below. The deviation was medium at large GSDs but then shrank to a very low level for small GSDs.

Antarctic Terns had a very low detection rate, only being detectable at all at a GSD of $30 \mathrm{~mm}$ and below. Even at the lowest tested GSD of $7 \mathrm{~mm}$, which was the finest ground resolution used in this study, the detection rate was lower than $60 \%$ with a high deviation.

Kelp Gulls showed an almost linear increase in detectability with decreasing GSD, reaching almost $100 \%$ detection rate with a medium deviation at all GSDs except for the largest. They were detectable at least with a 25-30\% rate even at large GSDs.

As Antarctic Terns, Skuas had a very low detection rate, only reaching $50 \%$ at the smallest tested GSD. The deviation was medium. 
For Snowy Sheathbills, the detection rate increased again almost linear, starting at about $25 \%$ in $80 \mathrm{~mm}$ GSD reaching about $90 \%$ at the smallest tested GSD. The deviation was higher in intermediate GSDs than in the largest/smallest GSD.

Adult Southern Giant Petrels started with a $0 \%$ detection rate at the highest tested GSD $(90 \mathrm{~mm})$ and increased to almost $100 \%$ at $10 \mathrm{~mm}$. The deviation was again medium in intermediate GSDs while almost zero at the largest/smallest tested GSD. Juvenile individuals had a lower detection rate at large GSDs, but also reached almost $100 \%$ at small GSDs. Here, even in the smallest GSD, the deviation was relatively strong.

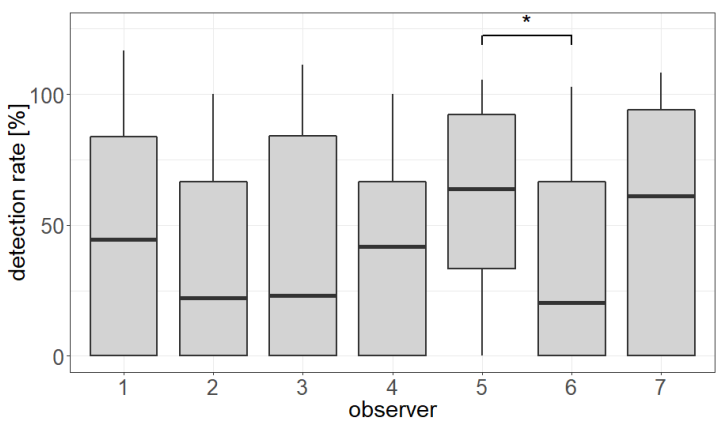

Figure 5. Comparison of the detection rate accomplished by the different observers. Only two observers were found to have statistically different detection rates (marked with $*$ )

\begin{tabular}{|c|c|c|c|c|c|c|}
\hline Observer & $\mathbf{1}$ & $\mathbf{2}$ & $\mathbf{3}$ & $\mathbf{4}$ & $\mathbf{5}$ & $\mathbf{6}$ \\
\hline $\mathbf{2}$ & 1.000 & & & & & \\
\hline $\mathbf{3}$ & 1.000 & 1.000 & & & & \\
\hline $\mathbf{4}$ & 1.000 & 1.000 & 1.000 & & & \\
\hline $\mathbf{5}$ & 1.000 & 0.056 & 0.276 & 0.563 & & \\
\hline $\mathbf{6}$ & 1.000 & 1.000 & 1.000 & 1.000 & $\mathbf{0 . 0 2 4}$ & \\
\hline $\mathbf{7}$ & 1.000 & 0.716 & 1.000 & 1.000 & 1.000 & 0.507 \\
\hline
\end{tabular}

Table 5. Results of the Wilcoxon test comparing the different observers (1-7) for all GSDs and species pooled. Significant relations are given in bold.

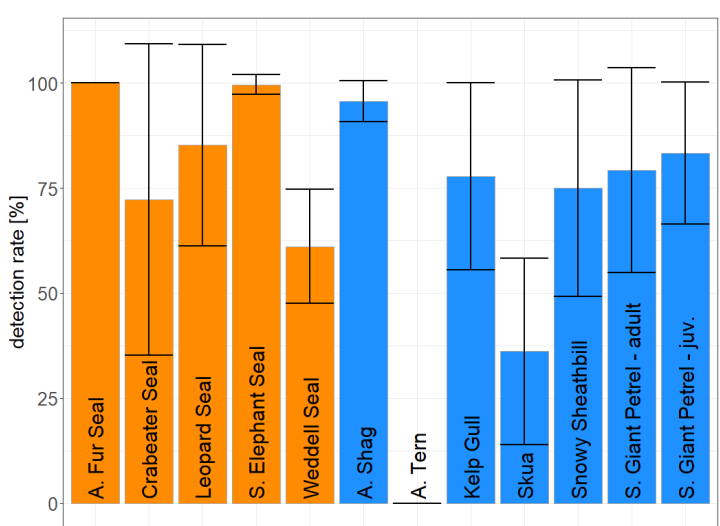

Figure 6. Detection rate of different species at a GSD of $30 \mathrm{~mm}$.

As can be seen in Figure 6, almost all species show at least a $50 \%$ detection rate at a GSD of $30 \mathrm{~mm}$. Only Skuas (about $40 \%$ detectability) and Antarctic Terns (not detectable) have a lower detection rate. Here, also the seal-category (all seals including those, where the observer could not identify the species) is depicted. As can be seen in the Figure 6, seals as such were detected in almost $100 \%$ of the cases. However, the species recognition within seals was lower, only Fur Seals and Elephant Seals were recognized with high certainty (with some mismatches, Fig. 8). In birds, Antarctic Shag nests reached an equally high detection rate $(>90 \%)$, all other species (except the ones mentioned before) showed a detection rate of 75-80\%.

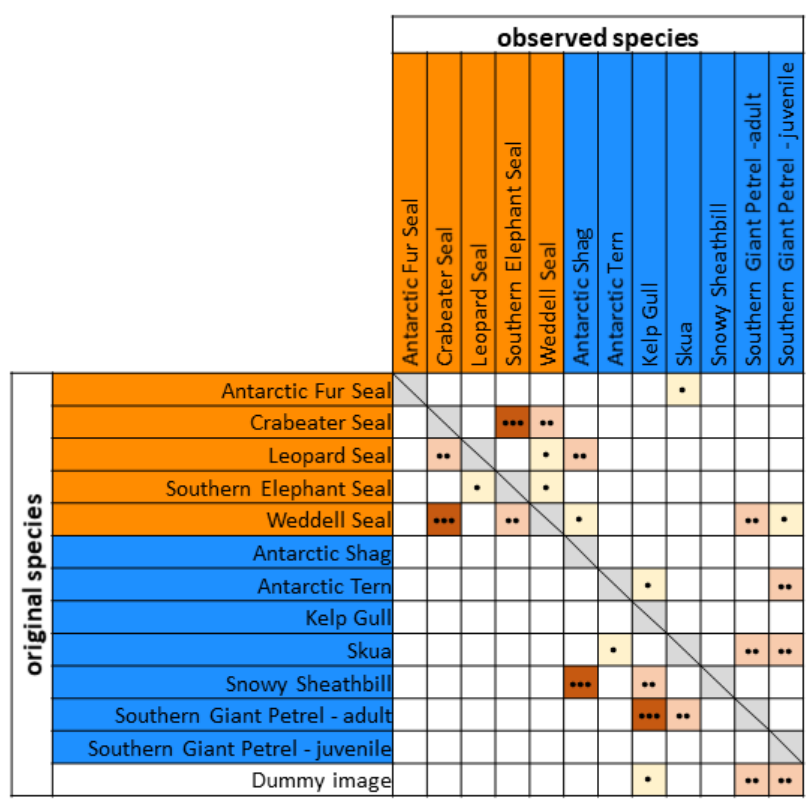

Figure 7. Confusion matrix. Lines show original species (to be found on the image), columns show observed species. Number of mismatches is given in three classes: $\bullet<3 ; \bullet \bullet 3-10 ; \bullet \bullet \bullet>10$ For example: In the first line Antarctic Fur Seals are pictured, but in 1-3 cases a Skua was counted by the observers.

Figure 7 shows the number of mismatches and between which species confusion occurred. It should be noted that a misassignment occurred not necessarily due to incorrect species recognition, but may be that other objects (e.g. stones) were incorrectly assigned to one of the species. This is particular the case for birds that were detected in seal or dummy images. In contrast, no seal was detected in a bird or dummy image. Most confusions happened between different seal species, except Fur Seals. In particular, Crabeater Seals and Weddell Seals were frequently confused with each other and with Southern Elephant Seals. For the birds most confusion happened to images with Snowy Sheathbills (with Antarctic Shag and Kelp Gull) and to those with adults of Southern Giant Petrels (with Kelp Gull and Skua). Occasionally adults and juveniles of Southern Giant Petrel were detected incorrectly in pictures with Skuas. Antarctic Shags, Kelp Gulls and juvenile Southern Giant Petrels in the original image were never confused with another species.

\section{DISCUSSION}

Aim of this study is to support the planning and analysis of UAV campaigns to monitor seals and flying seabirds in the Antarctic. However, the results are subject to some constraints, which have to be considered regarding their representativeness: The investigations took place during austral summer in a small study area of the maritime Antarctic with the species frequently found there. Possible regional or temporal morphological differences could not be considered, as well as the confusion with not investigated Antarctic species (e.g. Cape Petrel (Daption capense), Snow Petrel (Pagodroma nivea), Ross Seal (Ommatophoca rossii)). It was shown that the results of the six observers were comparable. Nevertheless, it can be assumed that the experience of the observers with the respective species, both in the field and in aerial photo evaluation, is an important prerequisite for the analysis. 
It must be mentioned that the background of an aerial photograph does not play a role in distinguishing species, but it does play a role in finding an individual. In general, this is more difficult with complex topography and vegetation cover than with uniform substrates such as ice or rock, as they differ from the coloration of the respective species.

This study showed the importance of the GSD for detecting different species, however other factors affecting the detectability like the image quality (e.g. ISO speed, motion blur) or lightning conditions (e.g. cast shadows) were not considered.

\subsection{Detection characteristics}

The detection characteristics relate to body size measurements and the visual appearance of individuals at aerial images. Confusion between seals and birds seems unlikely due to the sheer body sizes. The body size in aerial images strongly depends on the posture of the animals and is therefore expected to vary more than anatomical measurements.

For seals the most distinguishable species is the Antarctic Fur Seal. Its body size is unique, as well as its physical appearance in the image. In the case of other seal species, the distinction is less clear, so that some confusion is possible. If body lengths over $3.5 \mathrm{~m}$ are measured, however, it is highly probable that these are Southern Elephant Seals. By contrast, body lengths between 1.8 and $2.3 \mathrm{~m}$ are likely to occur in Crabeater Seals. The body measurements of Leopard Seals, Weddell Seals, smaller Elephant Seals and larger Crabeater Seals overlap. These species are also visually more similar. How clearly the distinguishing features stand out also depends on the image situation (curved position, visibility of the flippers, head posture, group).

In terms of body sizes of birds, there are overlaps between Antarctic Tern and Snowy Sheathbill, with the former tending to be slimmer and somewhat shorter. Optical indicators such as the distinctive black cap and bifid tail of the Antarctic Terns make these species well distinguishable. Although this species is easy to identify, it is often not easy to spot it in particular on an underground that is complex in color and structure which is usually the case in their breeding habitat.

Even though many Skuas appear slightly larger than Kelp Gulls in aerial images, there is a clear overlap in the range of visible body dimensions. In the coloring, however, both species are well distinguishable from each other. Confusion of Skuas with relatively small individuals of Southern Giant Petrel is more likely, but still in most cases body measurements are a good aid to distinguish between both species. Occasionally visible characteristics, like the long neck and large beak of the Giant Petrels add in. Skuas and Giant Petrels are visually well adapted to their underground. While this can be compensated by the size of Giant Petrels, Skuas are often overlooked even in highresolution images. Depending on their age, juveniles of Southern Giant Petrels are well detectable, too. This is mainly due to their, compared to other species, large size and their fairly uniform plumage. This makes it possible to monitor the breeding success of a colony besides the population size.

Due to the irregular shape of individual Antarctic Shags in aerial images they are easiest to recognize by their nests. Shape and color of the nests and the mean distance between them makes colonies of this species clearly distinguishable from other objects or colonies of other species (e.g. penguins). To these nests thereupon mostly also an individual can be assigned.

\subsection{Appropriate Ground Sample Distance (GSD)}

The comparison of the observers showed that the individual observer has a low impact on the detection rate. Except for one significant relation there were no differences between observers. All observers included in the study were highly experienced in detecting the species in the field and at least experienced in detecting animals on UAV images. The results will certainly be different for inexperienced observers, but since monitoring of Antarctic species is expected to be accomplished by experienced scientists, this group will not be discussed here.

The comparison of the detection rate at different GSDs revealed different appropriate thresholds for different species (Figure 8). It is important to notice that there can be no clear distinction made by this approach between the detectability (finding the individual) and correctly addressing the species.

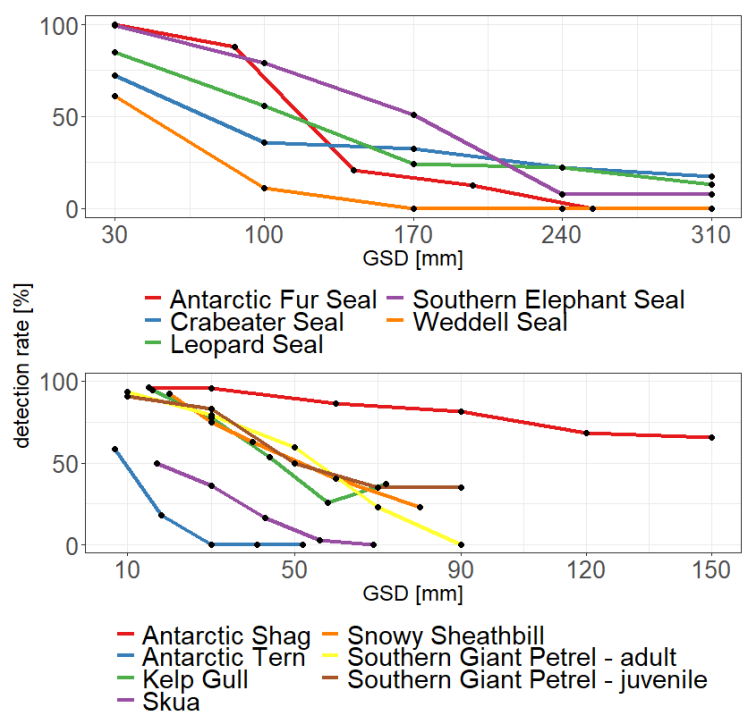

Figure 8. Comparison of mean detection rates at different GSDs for the species tested in the study

Almost all seals reached a relatively high level of detection of at least $75 \%$. For Fur Seals and Elephant Seals even a $100 \%$ detection rate can be accomplished by an adequate ground resolution. In Crabeater Seals and Leopard Seals the deviation was relatively high, leading to the interpretation that there is a high amount of uncertainty between observers in these species. On the other hand in species with low deviation like Elephant Seals, Fur Seals or Weddell Seals, all observers reached similar results. Weddell Seals reached the lowest detection rates of all seals independent of the observers, meaning that there is either a low detectability or a high amount of confusions with other species (see following paragraph on mismatches). It can be concluded that in a study using the described GSDs, conductors can be relatively sure of their counting results in the mentioned species with low deviation, whereas for the uncertain species Crabeater Seal and Leopard Seal the result will be more depending on the experience of the observer.

In birds, the tested GSDs were in a lower range, accounting for the smaller size of birds in comparison to seals. A high detection rate was reached by Antarctic Shag nests (even at very large GSDs) and Snowy Sheathbills, Southern Giant Petrels and Kelp Gulls at least in small GSDs. Those species are easily detectable due to their size or conspicuous appearance. Antarctic Terns were very hard to detect even at very small (and though practically almost not realizable) GSDs due to their very small size and good camouflage. Almost as hard to detect were Skuas, achieving only $50 \%$ detectability even at a small GSD. Here, the size is not as critical, since the same-sized Kelp Gull has a much better detection rate, but again there is a very good disguise of the animals against the environment, making it hard to find them. 
At a GSD of $30 \mathrm{~mm}$, seals are clearly detectable, but distinguishing between species is still not absolutely certain in all cases. For Antarctic Fur Seals and Southern Elephant Seals, monitoring is possible at this GSD with good certainty as well as counting seals without species assessment. In birds, this GSD allows to relatively safely identify nests of Antarctic Shag and to detect other bird species with at least $75 \%$ detection rate. Skuas and Antarctic Terns cannot be detected reliably at this GSD.

The analysis of detection mismatches cannot differentiate whether the mismatch occurred due to confusion between the considered species or with another object. The latter is likely the case for all birds observed in seal images due to the very different body sizes. Similarly, the false detection of Antarctic Shags in Snowy Sheathbill images with certainty is caused by nesting penguins in the images, which are easily to be confused with Shag nests. Wrongly detected adults and juveniles of Southern Giant Petrels appear to be the most likely source of confusion with other species or objects. Kelp Gulls and juvenile Southern Giant Petrels are well detectable but bear the risk of overinterpretation. Generally, the data shows that more confusion happens in seals than in birds, excluding the Fur Seals which bear a low risk of confusion.

\section{CONCLUSIONS}

The central idea of this study is to provide, beyond feasibility and case studies, the basis for an operational implementation of UAV-based mappings of Antarctic wildlife and to enable a comparability of the results of different campaigns. The distinction between different species of seals and flying seabirds is examined as well as the detection rate depending on the ground sample distance (GSD). A concise summary of the results is given in Table 6 . This can be used before a campaign to support flight planning and also as a mapping guide for the analysis of the captured aerial photographs. Future research should consider an enlargement of the database of individuals used for this study regarding spatial and temporal variability, the influence of the underground and the incorporation of further species. This study focuses on the detection of individuals. An extension of the methodology to include population parameters such as breeding pair numbers and breeding success would be a further important step towards the operationalization and standardization of UAV-based surveys of Antarctic wildlife.

\section{REFERENCES}

Adame, K., Pardo, M.A., Salvadeo, C., Beier, E., ElorriagaVerplancken, F.R., 2017. Detectability and categorization of California sea lions using an unmanned aerial vehicle. Mar. Mammal Sci. 33, 913-925.

Borowicz, A., McDowall, P., Youngflesh, C., Sayre-McCord, T., Clucas, G., Herman, R., Forrest, S., Rider, M., Schwaller, M., Hart, T., Jenouvrier, S., Polito, M.J., Singh, H., Lynch, H.J., 2018. Multi-modal survey of Adélie penguin mega-colonies reveals the Danger Islands as a seabird hotspot. Sci. Rep. 8, 3926.

Chabot, D., Craik, S.R., Bird, D.M., 2015. Population Census of a Large Common Tern Colony with a Small Unmanned Aircraft. PLOS ONE 10, e0122588.

Environmental Systems Research Institute - ESRI, 2016. ArcGIS Release 10.5. Redlands, CA.
Goebel, M.E., Perryman, W.L., Hinke, J.T., Krause, D.J., Hann, N.A., Gardner, S., LeRoi, D.J., 2015. A small unmanned aerial system for estimating abundance and size of Antarctic predators. Polar Biol. 38, 619-630.

Hodgson, J.C., Baylis, S.M., Mott, R., Herrod, A., Clarke, R.H., 2016. Precision wildlife monitoring using unmanned aerial vehicles. Sci. Rep. 6, 22574.

Hodgson, J.C., Mott, R., Baylis, S.M., Pham, T.T., Wotherspoon, S., Kilpatrick, A.D., Raja Segaran, R., Reid, I., Terauds, A., Koh, L.P., 2018. Drones count wildlife more accurately and precisely than humans. Methods Ecol. Evol. 9, $1160-1167$.

Korczak-Abshire, M., Zmarz, A., Rodzewicz, M., Kycko, M., Karsznia, I., Chwedorzewska, K.J., 2018. Study of fauna population changes on Penguin Island and Turret Point Oasis (King George Island, Antarctica) using an unmanned aerial vehicle. Polar Biol.

Krause, D.J., Hinke, J.T., Perryman, W.L., Goebel, M.E., LeRoi, D.J., 2017. An accurate and adaptable photogrammetric approach for estimating the mass and body condition of pinnipeds using an unmanned aerial system. PLOS ONE 12, $\mathrm{e} 0187465$.

Mustafa, O., Esefeld, J., Grämer, H., Maercker, J., Rümmler, M.-C., Pfeifer, C., 2017. Monitoring penguin colonies in the Antarctic using remote sensing data. On behalf of the German Environment Agency. Dessau-Roßlau.

Perryman, W., Goebel, M.E., Ash, N., LeRoi, D., Gardner, S., 2014. Small unmanned aerial systems for estimating abundance of krill-dependent predators: A feasibility study with preliminary results. AMLR 2011, 64-72.

Pfeiffer, S., 2005. Effects of Human Activities on Southern Giant Petrels and Skuas in the Antarctic. University Jena, Jena.

R Development Core Team (2018). R: A language and environment for statistical computing. $\mathrm{R}$ Foundation for Statistical Computing, Vienna, Austria.

Ratcliffe, N., Guihen, D., Robst, J., Crofts, S., Stanworth, A., Enderlein, P., 2015. A protocol for the aerial survey of penguin colonies using UAVs. J. Unmanned Veh. Syst. 3, 95-101.

Rümmler, M.-C., Mustafa, O., Maercker, J., Peter, H.-U., Esefeld, J., 2018. Sensitivity of Adélie and Gentoo penguins to various flight activities of a micro UAV. Polar Biol.

Savazzi, E., 2011. Digital photography for science: close-up photography, macrophotography and photomacrography. Lulu.com, Raleigh, NC.

Schuster, K.C., 2010. Impact of Human and Other Disturbance on Behaviour and Heart Rate of Incubating Adélie Penguins (Pygoscelis adeliae). Philips-Universität Marburg, Marburg.

Walker, G., 2014. Augmenting Steller Sea Lion Surveys in The Western Aleutians with Unmanned Aircraft. Proj. Number 1120 Fairbanks AK. 


\section{APPENDIX}

\begin{tabular}{|c|c|c|c|c|c|}
\hline & $\begin{array}{c}\text { Antarctic Fur Seal } \\
\text { Arctocephalus } \\
\text { gazella }\end{array}$ & $\begin{array}{l}\text { Crabeater Seal } \\
\text { Lobodon } \\
\text { carcinophagus }\end{array}$ & $\begin{array}{c}\text { Leopard Seal } \\
\text { Hydrurga } \\
\text { leptonyx } \\
\end{array}$ & $\begin{array}{l}\text { Southern Elephant Seal } \\
\text { Mirounga leonina }\end{array}$ & $\begin{array}{c}\text { Weddell Seal } \\
\text { Leptonychotes } \\
\text { weddelli }\end{array}$ \\
\hline Length & $1.09-1.50$ & $2.06-2.30$ & $2.64-3.08$ & $2.51-3.73$ & $2.30-2.70$ \\
\hline Width [m] & $0.38-0.48$ & $0.48-0.56$ & $0.57-0.69$ & $0.58-1.00$ & $0.58-0.71$ \\
\hline Description & $\begin{array}{l}\text { Colorful: brown, } \\
\text { blonde, black, grey; } \\
\text { smallest seal in the } \\
\text { Antarctic; hind } \\
\text { flippers are mostly } \\
\text { not visible, as they } \\
\text { are rotated forward; } \\
\text { head often turned to } \\
\text { the side }\end{array}$ & $\begin{array}{l}\text { Color variably: } \\
\text { brown, blonde, } \\
\text { creamy, grey, silver- } \\
\text { white; usually } \\
\text { brighter than other } \\
\text { seals, body relatively } \\
\text { slender }\end{array}$ & $\begin{array}{l}\text { Grey, dorsal } \\
\text { almost black, } \\
\text { large head, } \\
\text { usually stretched, } \\
\text { cigar-like, often } \\
\text { at floating ice, } \\
\text { appears a little } \\
\text { stiff }\end{array}$ & $\begin{array}{l}\text { Color variably: dark to pale } \\
\text { grey, brown, blotchy during } \\
\text { moult; relatively rotund } \\
\text { body shape; head end often } \\
\text { appears cigar-shaped } \\
\text { pointed; adult males larger } \\
\text { than all other seals; } \\
\text { immature like females; often } \\
\text { close together in groups }\end{array}$ & $\begin{array}{l}\text { Grey, ventral } \\
\text { brighter, small } \\
\text { head, small } \\
\text { flippers, rotund } \\
\text { body shape, often } \\
\text { with bend } \\
\text { posture }\end{array}$ \\
\hline $\begin{array}{l}\text { Confusion } \\
\text { risk }\end{array}$ & 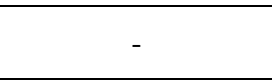 & Weddell Seal & $\begin{array}{l}\text { Weddell Seal, } \\
\text { Crabeater Seal }\end{array}$ & Weddell Seal & $\begin{array}{l}\text { Leopard Seal, } \\
\text { Crabeater Seal }\end{array}$ \\
\hline $\begin{array}{l}\text { GSD [mm] } \\
\text { (min/recom.) }\end{array}$ & $86 / 86$ & $30 /<30$ & $100 / 30$ & $170 / 30$ & $30 /<30$ \\
\hline
\end{tabular}

\begin{tabular}{|c|c|c|c|c|c|c|}
\hline & $\begin{array}{c}\text { Antarctic Shag } \\
\text { Phalacrocorax } \\
\text { atriceps }\end{array}$ & $\begin{array}{l}\text { Antarctic Tern } \\
\text { Sterna vittata }\end{array}$ & $\begin{array}{l}\text { Kelp Gull } \\
\text { Larus } \\
\text { dominicanus }\end{array}$ & $\begin{array}{c}\text { Skua } \\
\text { Catharacta spec. }\end{array}$ & $\begin{array}{c}\text { Snowy } \\
\text { Sheathbill } \\
\text { Chionis alba }\end{array}$ & $\begin{array}{l}\text { S. Giant Petrel } \\
\text { Macronectes } \\
\text { giganteus }\end{array}$ \\
\hline Length & $0.42-0.54$ & $0.25-0.30$ & $0.42-0.50$ & $0.45-0.55$ & $0.27-0.33$ & $0.53-0.68$ \\
\hline Width [m] & $1.11-1.65^{*}$ & $0.11-0.13$ & $0.20-0.25$ & $0.21-0.30$ & $0.13-0.15$ & $0.28-0.32 * *$ \\
\hline Description & $\begin{array}{l}\text { Nests are distinct } \\
\text { circular with star- } \\
\text { shaped guano } \\
\text { stains; usually at } \\
\text { rocks and cliffs; } \\
\text { adult individuals } \\
\text { appear irregular } \\
\text { black and white; } \\
\text { juveniles brown }\end{array}$ & $\begin{array}{l}\text { Greyish and } \\
\text { white; black } \\
\text { cap and deeply } \\
\text { forked tail are } \\
\text { distinctive }\end{array}$ & $\begin{array}{l}\text { White head and } \\
\text { tail; dorsal slate- } \\
\text { black back often } \\
\text { with a white } \\
\text { mark close to the } \\
\text { tail; curved } \\
\text { boundary } \\
\text { between head } \\
\text { and back } \\
\text { coloring; in fine } \\
\text { resolution the } \\
\text { yellow bill can } \\
\text { be visible }\end{array}$ & $\begin{array}{l}\text { Well } \\
\text { camouflaged; } \\
\text { heterogeneous in } \\
\text { color and } \\
\text { pattern; greyish- } \\
\text { brown in } \\
\text { different } \\
\text { variations; tail } \\
\text { and head mostly } \\
\text { darker than the } \\
\text { back }\end{array}$ & $\begin{array}{l}\text { Entirely white } \\
\text { with slight } \\
\text { shades of grey; } \\
\text { distinct drop- } \\
\text { shaped; often } \\
\text { in colonies of } \\
\text { other birds }\end{array}$ & $\begin{array}{l}\text { Adults: mostly } \\
\text { heterogeneous } \\
\text { greyish-brown; head, } \\
\text { neck and breast often } \\
\text { brighter or white; } \\
\text { completely dark or } \\
\text { white morphs } \\
\text { possible; large pale } \\
\text { yellowish bill; } \\
\text { Juveniles: gray with } \\
\text { lighter head; } \\
\text { roundish; fluffy } \\
\text { appearance }\end{array}$ \\
\hline $\begin{array}{l}\text { Confusion } \\
\text { risk }\end{array}$ & Penguins & $\begin{array}{c}\text { Snowy } \\
\text { Sheathbill }\end{array}$ & 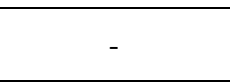 & S. Giant Petrel & Antarctic Tern & Skua \\
\hline $\begin{array}{l}\begin{array}{l}\text { GSD [mm] } \\
(\mathrm{min} / \text { recom.) }\end{array} \\
\end{array}$ & $120 / 90$ & $70 /<70$ & $44 / 16$ & $17 /<17$ & $40 / 20$ & $\begin{array}{c}50 / 10 \\
(50 / 30) \\
\end{array}$ \\
\hline
\end{tabular}

* nest diameter/nest distance; ** ‘length and width’ for juvenile Giant petrels not suitable
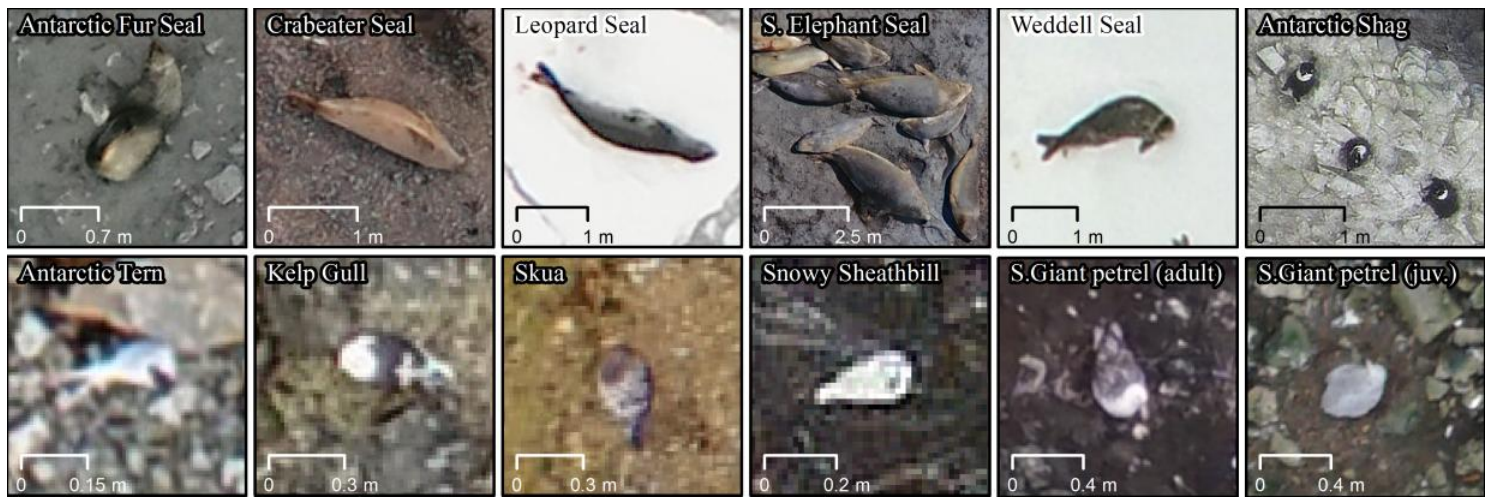

This guide is intended to support the identification of seals and birds in aerial images based on this study. All data refer to the visual appearance of the individuals at aerial images. 'Length/Width' corresponds to the mean value added/subtracted with the standard deviation. Unlike the other species the data for Antarctic Shags do not refer to the body of the animal but to diameter and distance of the nests. Body sizes for juvenile Giant Petrels are not mentioned, as they vary with the age of the individuals. The GSD specifications are intended to provide information to support an appropriate mission planning. The mentioned GSD values refer to detection rates of $50 \%$ (minimum) and $80 \%$ (recommended) according to this study. 